\title{
Changes of Plasma Adiponectin Levels after Smoking Cessation
}

\author{
Wang-Youn Won, Chang-Uk Lee, Jeong-Ho Chae, Jung-Jin Kim, Chul Lee, and Dai-Jin Kim $\bowtie$ \\ Department of Psychiatry, Seoul St. Mary's Hospital, The Catholic University of Korea College of Medicine, Seoul, Republic of Korea
}

\begin{abstract}
Objective Cigarette smoking is associated with a variety of health problems including cardiovascular, pulmonary, neoplasms, endocrinopathies including diabetes, the metabolic syndrome, and chronic inflammation. Adiponectin is an adipocyte-derived plasma protein that is closely associated with insulin sensitivity and the metabolic syndrome. The aim of this study was to evaluate the changes of plasma adiponectin levels after smoking cessation.

Methods Thirty seven smokers that wanted to stop smoking without any nicotine replacement therapy or medication were recruited for this study. Fifteen smokers succeeded in stopping smoking (validated by urine cotinine levels $\leq 50 \mathrm{ng} / \mathrm{mL}$ ) and $22 \mathrm{smokers} \mathrm{failed.}$ Therefore, only the 15 that succeeded were included in the analysis. The plasma adiponectin levels were determined using a commercially available enzyme-linked immunosorbent assay.

Results The mean age of the successful 15 was $35 \pm 9.3$ years old. They were all males. The daily smoking habit was a mean of $13.5 \pm 5.4$ cigarettes per day. The mean Nicotine Dependence Syndrome Scale (NDSS) and Fagerstrom Test for Nicotine Dependence (FTND) scores were $55.6 \pm 9.6$ and 2.9 \pm 1.9. During the study period of three months, the mean body mass index (BMI), body fat mass (BFM), waist-hip ratio (WHR) and body weight increased by $1.1 \mathrm{~kg} / \mathrm{m}^{2}, 3.0 \%, 0.02 \%$, and $2.9 \mathrm{~kg}$, respectively. The baseline mean adiponectin level in the subjects was $11.9 \pm 5.2 \mathrm{mg} / \mathrm{L}$. The mean adiponectin levels measured at one and three months were $16.0 \pm 5.1 \mathrm{mg} / \mathrm{L}$ and $14.7 \pm 4.5 \mathrm{mg} / \mathrm{L}$ respectively. The mean plasma adiponectin levels of the successful group was significantly increased after four weeks when compared to the baseline $(\mathrm{z}=-2.401, \mathrm{p}=0.016)$. However, the decrease in plasma adiponectin levels at one and three months was not statistically significant.
\end{abstract}

Conclusion Even though the decrease over the next two months was not significant, these findings, the increase of plasma level of adiponectin after smoking cessation, provide preliminary data for future research on the possible mechanisms associated with smoking cessation and changes in body metabolism.

Psychiatry Investig 2014;11(2):173-178

Key Words Smoking cessation, Nicotine, Tobacco, Adiponectin, Ghrelin, Leptin, Metabolism.

\section{INTRODUCTION}

Cigarette smoking is associated with a variety of health problems including cardiovascular, pulmonary, neoplasms, endocrinopathies including diabetes, the metabolic syndrome, and chronic inflammation. ${ }^{1}$ Among the many toxic materials existing in cigarettes, nicotine is the primary addictive substance. Nicotine dependence is a growing health problem worldwide. Many investigators are studying methods to reduce the preva-

Received: March 8, 2013 Revised: August 26, 2013

Accepted: September 2, 2013 Available online: April 11, 2014

$\triangle$ Correspondence: Dai-Jin Kim, MD, PhD

Department of Psychiatry, Seoul St. Mary's Hospital, The Catholic University of Korea College of Medicine, 222 Banpo-daero, Seocho-gu, Seoul 137-701, Republic of Korea

Tel: +82-2-2258-6086, Fax: +82-2-594-3870, E-mail: kdj922@chollian.net

(a) This is an Open Access article distributed under the terms of the Creative Commons Attribution Non-Commercial License (http://creativecommons.org/licenses/by$\mathrm{nc} / 3.0$ ) which permits unrestricted non-commercial use, distribution, and reproduction in any medium, provided the original work is properly cited. lence of nicotine dependence.

Adiponectin is an adipocyte-derived plasma protein that is closely associated with insulin sensitivity and the metabolic syndrome., ${ }^{2,3}$ It has anti-inflammatory properties, inhibits the proliferation of vascular smooth muscle cells and suppresses the conversion of macrophages to foam cells. ${ }^{4}$ Low levels of plasma adiponectin have been reported to be associated with the risk for myocardial infarction or coronary heart disease. ${ }^{5,6}$ Smoking has been associated with low levels of plasma adiponectin in both healthy subjects and patients with coronary heart disease. ${ }^{7,8}$ Efstathiou et al. ${ }^{9}$ reported that smoking cessation increased serum adiponectin levels after two months in a healthy Greek population. Takefuji et al. ${ }^{10}$ reported that current smoking habits were associated with low adiponectin levels, and that there was a dose-dependent association between smoking intensity and adiponectin levels in current smokers.

However, there is no prior study on the serial changes of plasma adiponectin levels after smoking cessation. In addition, most 
of previous researches were designed as cross-sectional and used nicotine replacement therapy or medications. The aim of this study was to evaluate the changes of plasma adiponectin levels after smoking cessation. The subjects received no nicotine replacement therapy or medications (e.g., varenicline and bupropion). Moreover, the level of ghrelin and leptin, which have been associated with body weight and metabolism were assessed to determine their relationship to smoking cessation.

\section{METHODS}

Thirty seven smokers that wanted to quit smoking were recruited from the Bucheon St. Mary's Hospital, the Catholic University of Korea. All subjects fulfilled the DSM-IV diagnostic criteria for the current diagnosis of nicotine dependence. ${ }^{11}$ The DSM-IV diagnoses were determined in a consensus with two board certificated psychiatrists using all available clinical instruments, including a semi-structured interview based on the DSM-IV criteria. The demographic data was collected at baseline. The levels of nicotine dependence were assessed using the Korean version of the Nicotine Dependence Syndrome Scale (NDSS) and the Fagerstrom Test for Nicotine Dependence (FTND). ${ }^{12}$ The exclusion criteria included current or past history of comorbid psychiatric illness, any neurological disorder, significant medical conditions, abnormal results on laboratory screening tests, addiction to alcohol or substances other than nicotine, and a history of head trauma accompanied by a loss of consciousness. All the participants agreed to quit smoking without any nicotine replacement therapy or nicotine-related medications, and to participate in a urine cotinine monitoring schedule over three months.

During the study period, smoking cessation was evaluated by both a self-report questionnaire and urine cotinine levels. The urine cotinine levels of all subjects were monitored at baseline and after 3 months. Urinary samples were taken without prior notification. The urine specimens were collected in a plastic container and stored at $4^{\circ} \mathrm{C}$. The assay was performed on the day the sample was collected. Cotinine was analyzed using the Cotinine Enzyme Immunoassay on a 502X Multiple Chemistry Unit automated analyzer. The assay procedures and quality control were carried out according to the manufacturer's instructions. Briefly, $35 \mu \mathrm{L}$ of urine or calibrator was added in 125 $\mu \mathrm{L}$ of reagent including cotinine specific monoclonal antibodies and enzyme substrate. Then, $125 \mu \mathrm{L}$ of cotinine labeled enzyme, and glucose-6-phosphate dehydrogenase was dispensed and incubated at $37^{\circ} \mathrm{C}$. Spectrophotometric measurement was performed at $340 \mathrm{~nm}$. Among the 37 participants, 15 smokers succeeded in quitting smoking (validated by urine cotinine lev$\mathrm{el} \leq 50 \mathrm{ng} / \mathrm{mL}$ ) and 22 smokers failed. Therefore, the 15 successful participants were included in the analysis.
The purpose and entire protocol of the study were explained to all subjects. All subjects provided written informed consent. The study protocol was approved by the hospital ethics committee of the Bucheon St. Mary's Hospital, the Catholic University of Korea. All the procedures followed were in accordance with the Helsinki Declaration of 1975, as revised in 1983.

Body composition was analyzed using the Inbody 720 (Biospace CO., Ltd., Seoul, Korea). All body composition parameters such as body mass index (BMI), body fat mass (BFM) and waist hip ratio (WHR) at baseline and after abstinence were included in the analysis.

Blood samples for routine blood chemistry were obtained from the participants four times: at baseline, and after 1,2, and 3 months. Blood was drawn from all subjects at 7:00 a.m. after an overnight fasting.

The plasma adiponectin levels were checked at baseline, 1 , and 3 months after smoking cessation. The plasma adiponectin levels were determined using a commercially available enzyme-linked immunosorbent assay (Human Adipokine Panel A Multiplex Immunoassay Kit - ONE PLEX cat No. HADK161K-A01). The plasma leptin levels were determined using a radioimmunoassay (Linco Research, St. Charles, MO, USA) that utilizes ${ }^{125}$-labeled bioactive human leptin as a tracer as well as a rabbit polyclonal antibody against human leptin. The plasma ghrelin levels were measured using a commercially available RIA kit (Phoenix Pharmaceuticals, Inc., Belmont, CA, USA) that utilizes ${ }^{125}$ I-labeled bioactive ghrelin as a tracer and a rabbit polyclonal antibody against the full-length octanoylated human ghrelin.

The Kolmogorov-Smirnov test did not show a normal distribution of the data. Therefore, non-parametric tests were used to evaluate statistical significance. A Wilcoxon signed rank test was used to analyze the differences between baseline and at each point in time that the plasma adiponectin levels were obtained in the subjects. The Spearman's correlation test was carried out to analyze the correlation between the plasma levels of adiponectin and the levels of ghrelin, leptin or other variables. All of the statistical analyses were carried out using the Statistical Package for the Social Sciences (SPSS) version 12.0 for Windows. The level of statistical significance was set at $p<0.05$. All data are presented as the mean \pm standard deviation (SD).

\section{RESULTS}

The demographic data at baseline were as follows: the mean age of the successful 15 was $34 \pm 8.4$ years of aged. They were all males. The daily cigarettes (cigarettes/day) smoked were 12.9 \pm 5.3 . The mean NDSS and FTND scores were 55.9 \pm 9.2 and $2.8 \pm 1.9$.

All of the body composition parameters such as BMI, BFM, 
WHR and weight were higher in the 15 subjects after 3-months of abstinence when compared to the baseline (Table 1). After the 3 month period of smoking cessation, the mean BMI, BFM, WHR and body weight increased by $1.1 \mathrm{~kg} / \mathrm{m}^{2}, 3.0 \%, 0.02 \%$, and $2.9 \mathrm{~kg}$, respectively.

Figure 1 shows the plasma adiponectin levels at each point in time for the 15 successful participants. The baseline adiponectin level was $11.9 \pm 5.2 \mathrm{mg} / \mathrm{L}$. The mean adiponectin levels measured at 1 month and 3 months were $16.0 \pm 5.1 \mathrm{mg} / \mathrm{L}$ and $14.7 \pm 4.5 \mathrm{mg} / \mathrm{L}$. The mean plasma adiponectin levels of the quitters were significantly increased after 4 weeks when compared to baseline $(\mathrm{z}=-2.401, \mathrm{p}=0.016)$. However, the decrease in plasma adiponectin level between month 1 and month 3 was not statistically significant $(\mathrm{z}=-0.801, \mathrm{p}=0.423)$.

The plasma level of leptin increased and level of ghrelin decreased after smoking cessation although the changes of vari- ables are not significant. There was no significant correlation between adiponectin and ghrelin or leptin, and the body composition indices analyzed. Regarding the lipid profiles including total cholesterol, HDL-cholesterol, LDL-cholesterol and triglyceride, all the variables increased after 4 weeks of smoking cessation.

\section{DISCUSSION}

The results of this study demonstrated that smoking cessation was significantly associated with increased plasma adiponectin levels. There are several reports with similar findings. Iwashima et al. ${ }^{13}$ and Takefuji et al. ${ }^{10}$ reported the association between smoking status and the plasma level of adiponectin. They showed that cigarette smoking correlateed with low plasma adiponectin levels. In addition, a dose-dependent associa-

Table 1. Demographic data and comparison of variables at each point in time

\begin{tabular}{|c|c|c|c|}
\hline Month & 0 & 1 & 3 \\
\hline Appetite (a) (VAS:cm) & $4.8 \pm 1.6$ & $5.6 \pm 1.9$ & $6.2 \pm 1.4^{*}$ \\
\hline Appetite (b) (VAS:cm) & $4.1 \pm 2.5$ & $4.3 \pm 2.3$ & $4.9 \pm 1.9^{*}$ \\
\hline Weight (kg) & $73.2 \pm 8.1$ & $74.2 \pm 7.6$ & $75.1 \pm 8.6^{*}$ \\
\hline $\mathrm{BMI}\left(\mathrm{kg} / \mathrm{m}^{2}\right)$ & $24.4 \pm 2.9$ & $24.9 \pm 2.7$ & $25.1 \pm 3.2^{*}$ \\
\hline Adiponectin (mg/L) & $11.9 \pm 5.2$ & $16.0 \pm 5.1^{*}$ & $14.7 \pm 4.5$ \\
\hline Leptin (ng/mL) & $4.8 \pm 1.1$ & $6.5 \pm 1.9$ & $7.6 \pm 2.3$ \\
\hline Ghrelin $(\mathrm{pg} / \mathrm{mL})$ & $129.2 \pm 23.6$ & $85.6 \pm 19.4$ & $71.3 \pm 10.5$ \\
\hline GOT (AST) (U/L) & $28.9 \pm 9.8$ & $31.4 \pm 11.6$ & $36.9 \pm 16.0$ \\
\hline GPT (ALT) (U/L) & $35.5 \pm 23.8$ & $38.9 \pm 30.2$ & $47.1 \pm 35.3$ \\
\hline Glucose (mg/dL) & $86.7 \pm 9.1$ & $86.5 \pm 10.2$ & $90.1 \pm 6.2$ \\
\hline Creatinine $(\mathrm{mg} / \mathrm{dL})$ & $1.0 \pm 0.1$ & $1.0 \pm 0.1$ & $1.0 \pm 0.1$ \\
\hline Uric acid (mg/dL) & $5.6 \pm 1.1$ & $6.0 \pm 1.2$ & $5.8 \pm 1.5$ \\
\hline Total cholesterol (mg/dL) & $182.8 \pm 33.3$ & $194.1 \pm 36.9$ & $180.0 \pm 32.1$ \\
\hline HDL-cholesterol (mg/dL) & $54.8 \pm 10.0$ & $56.9 \pm 10.4$ & $53.1 \pm 11.1$ \\
\hline LDL-cholesterol (mg/dL) & $114.6 \pm 29.6$ & $120.2 \pm 30.1$ & $108.6 \pm 27.7$ \\
\hline Triglyceride (mg/dL) & $111.7 \pm 81.1$ & $126.4 \pm 102.5$ & $150.9 \pm 93.6$ \\
\hline Insulin $(\mathrm{mcU} / \mathrm{mL})$ & $6.6 \pm 6.0$ & $9.7 \pm 8.5$ & $7.2 \pm 4.0$ \\
\hline WBC $\left(10^{9} / \mathrm{L}\right)$ & $6.9 \pm 1.1$ & $7.1 \pm 1.4$ & $7.0 \pm 1.0$ \\
\hline $\operatorname{RBC}\left(10^{12} / \mathrm{L}\right)$ & $5.2 \pm 0.4$ & $5.2 \pm 0.4$ & $5.2 \pm 0.4$ \\
\hline Hemoglobin (g/dL) & $15.7 \pm 1.2$ & $15.9 \pm 1.3$ & $15.8 \pm 1.2$ \\
\hline Hematocrit (\%) & $47.1 \pm 3.3$ & $47.1 \pm 3.5$ & $46.9 \pm 2.9$ \\
\hline Platelet $\left(10^{9} / \mathrm{L}\right)$ & $239 \pm 32.4$ & $241.27 \pm 33.4$ & $268.55 \pm 38.2$ \\
\hline MCV (fL) & $89.95 \pm 3.9$ & $89.47 \pm 2.3$ & $89.15 \pm 2.9$ \\
\hline $\mathrm{MCH}(\mathrm{pg})$ & $29.99 \pm 0.9$ & $30.15 \pm 0.8$ & $30.18 \pm 1.0$ \\
\hline $\mathrm{MCHC}(\%)$ & $33.36 \pm 1.0$ & $33.7 \pm 0.7$ & $33.86 \pm 1.0^{*}$ \\
\hline
\end{tabular}

*statistical significance between baseline and endpoint ( $\mathrm{p}<0.05)$. BMI: body mass index, AST: aspartate aminotransferase, ALT: alanine aminotransferase, HDL-cholesterol: high-density lipoprotein cholesterol, LDL-cholesterol: low-density lipoprotein cholesterol, WBC: white blood cell, RBC: red blood cell, MCV: mean corpuscular volume, $\mathrm{MCH}$ : mean corpuscular hemoglobin, MCHC: mean corpuscular hemoglobin concentration 


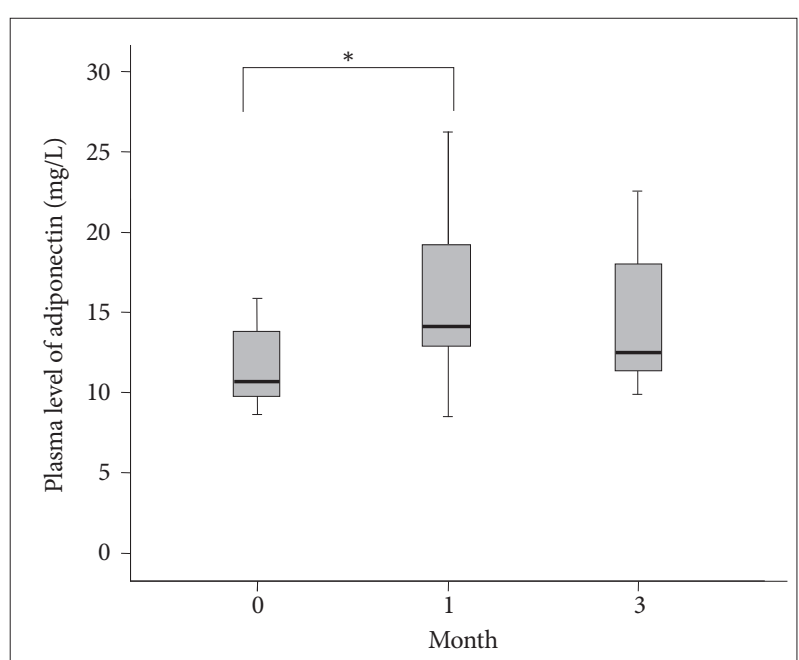

Figure 1. The box plots show the median and quartiles, and the whisker caps of the box plots show the mean 5th and 95th percentile values. ${ }^{*}$ indicates statistical significance $(p<0.05)$. A Wilcoxon signed rank test yielded the results. The plasma levels of adiponectin at baseline, 1 month, and 3 months were $11.9 \pm 5.2$, $16.0 \pm 5.1$, and $14.7 \pm 4.5 \mathrm{mg} / \mathrm{L}$, respectively. The mean plasma adiponectin levels were increased between baseline and 1 month $(z=-2.401, p=0.016)$, but decreased from month 1 to 3 months $(z=-0.801, p=0.423)$.

tion between smoking intensity and plasma adiponectin level in current smokers has been reported. ${ }^{10}$ Abbasi et al..$^{4}$ reported that there was a relationship between alterations in the plasma adiponectin concentration and insulin resistance in Caucassian smokers. Patel et al. ${ }^{15}$ reported that circulating serum adiponectin levels in patients with coronary artery disease were related to the atherosclerotic burden or risk. Most of the studies on the association between plasma levels of adiponectin and smoking status were cross-sectional studies. Recent research reported by Efstathiou et al. ${ }^{9}$ showed that serum adiponectin levels appeared to increase significantly within 2 months after smoking cessation.

To our knowledge, although the above studies showed an association between smoking cessation and plasma levels of adiponectin, serial changes in plasma adiponectin levels after smoking cessation have not been previously investigated. Furthermore, no nicotine replacement therapy or nicotine-related medications including varenicline or bupropion were used for smoking cessation in this study.

Adiponectin is known to improve insulin sensitivity and the lipid profile by stimulation of adenosine monophosphate-activated protein kinase in skeletal muscle cells and the liver. ${ }^{2}$ By contrast, insulin resistance which is related to low plasma levels of adiponectin, lower HDL-C concentration by stimulating the transcriptional activity of ApoAl, and by decreasing VLDL-C production, as well as by increasing the expression of lipoprotein lipase. ${ }^{2}$ Another example of the possible correlation between smoking and serum levels of adiponectin is the associa- tion of alterations of body fat distribution with the smoking of tobacco. ${ }^{16,17}$ A population-based cohort study showed that current smokers had higher WHR than non-smokers. ${ }^{18}$ Moreover, the smoking intensity (cigarettes per day) was positively correlated to the WHR in both males and females. ${ }^{19}$ The body composition and weight changes that occur after smoking cessation are explained by several mechanisms. Some of the proposed mechanisms include increased energy intake, decreased resting metabolic rate, decreased physical activity, and increased lipoprotein lipase activity. ${ }^{20-24}$

Leptin and ghrelin are thought to be related to appetite and energy metabolism. ${ }^{25,26}$ In our previous study, the relationship between smoking cessation and body metabolism including in the brain, was investigated. The findings showed that serum levels of leptin increased, and serum levels of ghrelin decreased after smoking cessation. ${ }^{27}$ Appetite has been shown to be regulated by an interaction between the central and peripheral nervous system, the leptin-ghrelin-neuropeptide Y feedback loop. The result of our prior study suggested that there are other mechanisms involved in the control of appetite after smoking cessation, in addition to leptin; this was based on the finding that leptin and appetite showed a negative correlation after smoking cessation. ${ }^{27}$ In the present study, we suggest that the adiponectin could play a part of role in these energy-appetite mechanisms.

Recently, adiponectin has been reported to generate a negative energy balance by increasing energy expenditure. ${ }^{28}$ In contrast to leptin, intracerebroventricular administration of adiponectin decreased body weight mainly by stimulating energy expenditure. Systemic adiponectin resulted in increased thermogenesis, weight loss and reduction in serum glucose and lipid levels. ${ }^{28}$ Adiponectin also potentiated the effect of leptin on thermogenesis and lipid levels. Adiponectin has unique central effects on energy homeostasis based on these findings. ${ }^{29}$ Spranger et al. reported that the identification of adiponectin receptors on brain endothelial cells and the finding of a modified secretion pattern of centrally active substances in bloodbrain barrier (BBB) cells provides an alternate explanation of how adiponectin effects energy metabolism..$^{28}$ In addition, Kubota et al. suggested that energy homeostasis may be mediated by both short-term regulators, such as gut hormones, and long-term regulators including adiponectin that allow energy to be stored efficiently. These findings offer an insight into adiponectin's influence on the central nervous system as well as the peripheral nervous system. ${ }^{30}$

The evidence suggests that there is an association between the body's energy metabolism, weight change, body composition, appetite-controlling gut hormones (ghrelin or leptin), and adiponectin, which all play a role in body metabolism and fat distribution. Even though medication effects associated with 
smoking cessation were excluded in this study, there were no correlations observed among the study variables. However, the plasma level of adiponectin increased within 4 weeks of smoking cessation and there was a decrease during the next 2 months, which was not statistically significant. This result may be due to another as yet to be identified mechanism associated with energy metabolism or due to one of the several limitations of this study.

The main limitation of this study was the small sample size, which did not have the statistical power to show serial changes in the plasma levels of adiponectin. The subjects that succeeded and were included in the analysis had lower FTND scores than the participants that could not be abstinent during the study period. If the heavy smokers were included, the results might have had a greater statistical power. The reasons of low FTND scores of participants and the low proportion of successful quitters (15 of 37) were because this study did not include using any nicotine replacement therapy, so the heavy smokers were not included in the analysis.

Another limitations are that plasma adiponectin levels of non quitters were not measured and the levels of smoking quitters after 2 months not checked in this study. Also, there are numbers of confounding factors affecting the adiponectin level.

In summary, although there are several limitations in the current study, the results of this study showed that the plasma level of adiponectin increased after a short period (4 weeks) of smoking cessation. Even though there was a decrease during the next 2 months, the difference was not statistically significant. These findings provide preliminary results for future research on the possible mechanisms associated with smoking cessation and the related changes in metabolism.

\section{Acknowledgments}

This study was supported by a grant of the Ministry of education, science and technology, 2009, Republic of Korea.

\section{REFERENCES}

1. US Department of Health and Human Services. The Health Consequences of Smoking: A Report of the Surgeon General. Rockville: US Department of Health and Human Services, Public Health Service, Office of the Surgeon General; 2004.

2. Beltowski J, Jamroz-Wisniewska A, Widomska S. Adiponectin and its role in cardiovascular diseases. Cardiovasc Hematol Disord Drug Targets 2008;8:7-46.

3. Matsuzawa Y, Funahashi T, Kihara S, Shimomura I. Adiponectin and metabolic syndrome. Arterioscler Thromb Vasc Biol 2004;24:29-33.

4. Havel PJ. Control of energy homeostasis and insulin action by adipocyte hormones: leptin, acylation stimulating protein, and adiponectin. Curr Opin Lipidol 2002;13:51-59.

5. Kumada M, Kihara S, Sumitsuji S, Kawamoto T, Matsumoto S, Ouchi $\mathrm{N}$, et al. Association of hypoadiponectinemia with coronary artery disease in men. Arterioscler Thromb Vasc Biol 2003;23:85-89.

6. Pischon T, Girman CJ, Hotamisligil GS, Rifai N, Hu FB, Rimm EB. Plasma adiponectin levels and risk of myocardial infarction in men.
JAMA 2004;291:1730-1737.

7. Miyazaki T, Shimada K, Mokuno H, Daida H. Adipocyte derived plasma protein, adiponectin, is associated with smoking status in patients with coronary artery disease. Heart 2003;89:663.

8. Thamer C, Stefan N, Stumvoll M, Hring H, Fritsche A. Reduced adiponectin serum levels in smokers. Atherosclerosis 2005;179:421-422.

9. Efstathiou SP, Skeva I, Dimas C, Panagiotou A, Parisi K, Tzanoumis L, et al. Smoking cessation increases serum adiponectin levels in an apparently healthy Greek population. Atherosclerosis 2009;205:632-636.

10. Takefuji S, Yatsuya H, Tamakoshi K, Otsuka R, Wada K, Matsushita K, et al. Smoking status and adiponectin in healthy Japanese men and women. Prev Med 2007;45:471-475.

11. American Psychiatric Association. Diagnostic and Statistical Manual of Mental Disorders, Fourth Edition. Washington, DC: American Psychiatric Association; 1994.

12. Heatherton TF, Kozlowsky LT, Frecker RC, Fagerstrom KO. The Fagerstrom test for nicotine dependence: a revision of the Fagerstrom tolerance questionnaire. Br J Addict 1991;86:1119-1127.

13. Iwashima Y, Katsuya T, Ishikawa K, Kida I, Ohishi M, Horio T, et al. Association of hypoadiponectinemia with smoking habit in men. Hypertension 2005;45:1094-1100.

14. Abbasi F, Farin HM, Lamendola C, McLaughlin T, Schwartz EA, Reaven GM, et al. The relationship between plasma adiponectin concentration and insulin resistance is altered in smokers. J Clin Endocrinol Metab 2006;91:5002-5007.

15. Patel JV, Abraheem A, Dotsenko O, Creamer J, Gunning M, Hughes EA, et al. Circulating serum adiponectin levels in patients with coronary artery disease: relationship to atherosclerotic burden and cardiac function. J Intern Med 2008;264:593-598.

16. Cnop M, Havel PJ, Utzschneider KM, Carr DB, Sinha MK, Boyko EJ, et al. Relationship of adiponectin to body fat distribution, insulin sensitivity and plasma lipoproteins: evidence for independent roles of age and sex. Diabetologia 2003;46:459-469.

17. Staiger H, Tschritter O, Machann J, Thamer C, Fritsche A, Maerker E, et al. Relationship of serum adiponectin and leptin concentrations with body fat distribution in humans. Obes Res 2003;11:368-372.

18. Canoy D, Wareham N, Luben R, Welch A, Bingham S, Day N, et al. Cigarette smoking and fat distribution in 21,828 British men and women: a population-based study. Obes Res 2005;13:1466-1475.

19. Bamia C, Trichopoulou A, Lenas D, Trichopoulos D. Tobacco smoking in relation to body fat mass and distribution in a general population sample. Int J Obes Relat Metab Disord 2004;28:1091-1096.

20. Dallosso HM, James WP. The role of smoking in the regulation of energy balance. Int J Obes 1984;8:365-375.

21. Ferrara CM, Kumar M, Nicklas B, McCrone S, Goldberg AP. Weight gain and adipose tissue metabolism after smoking cessation in women. Int J Obes Relat Metab Disord 2001;25:1322-1326.

22. Filozof C, Fernandez Pinilla MC, Fernndez-Cruz A. Smoking cessation and weight gain. Obes Rev 2004;5:95-103.

23. Moffatt RJ, Owens SG. Cessation from cigarette smoking: changes in body weight, body composition, resting metabolism, and energy consumption. Metabolism 1991;40:465-470.

24. Stamford BA, Matter S, Fell RD, Papanek P. Effects of smoking cessation on weight gain, metabolic rate, caloric consumption, and blood lipids. Am J Clin Nutr 1986;43:486-494.

25. Harvey J. Leptin regulation of neuronal excitability and cognitive function. Curr Opin Pharmacol 2007;7:643-647.

26. van der Lely AJ, Tschp M, Heiman ML, Ghigo E. Biological, physiological, pathophysiological, and pharmacological aspects of ghrelin. Endocr Rev 2004;25:426-457.

27. Lee H, Joe KH, Kim W, Park J, Lee DH, Sung KW, et al. Increased leptin and decreased ghrelin level after smoking cessation. Neurosci Lett 2006;409:47-51.

28. Spranger J, Verma S, Göhring I, Bobbert T, Seifert J, Sindler AL, et al. 
Adiponectin does not cross the blood-brain barrier but modifies cytokine expression of brain endothelial cells. Diabetes 2006;55:141-147.

29. Qi Y, Takahashi N, Hileman SM, Patel HR, Berg AH, Pajvani UB, et al. Adiponectin acts in the brain to decrease body weight. Nat Med 2004;
10:524-529.

30. Kubota N, Yano W, Kubota T, Yamauchi T, Itoh S, Kumagai H, et al. Adiponectin stimulates AMP-activated protein kinase in the hypothalamus and increases food intake. Cell Metab 2007;6:55-68. 